

\title{
HOSPITALES SUSTENTABLES: PARTIDAS CRÍTICAS PARA SU CONSTRUCCIÓN Y EL ROL DE LA INSPECCIÓN TÉCNICA
}

\author{
SUSTAINABLE HOSPITALS: CRITICAL ITEMS FOR THEIR \\ CONSTRUCTION AND THE ROLE OF TECHNICAL INSPECTION
}

\author{
PABLO CANALES \\ Magíster en Construcción Sustentable \\ Estudiante, Escuela de Construcción Civi \\ Pontificia Universidad Católica de Chile \\ Santiago, Chile \\ https://orcid.org/0000-0003-4096-9492 \\ pcanalesn@uc.cl
}

\author{
CLAUDIA VALDERRAMA-ULLOA \\ Doctor en Mecánica \\ Académico, Escuela de Construcción Civil \\ Pontificia Universidad Católica de Chile \\ Santiago, Chile \\ https://orcid.org/0000-0002-1603-9714 \\ c.valderrama@uc.cl
}

\author{
XIMENA FERRADA \\ Doctora en Ciencias de la Ingeniería \\ Directora carrera de Ingeniería Civil en Obras Civiles, \\ Facultad de Ingeniería Universidad del Desarrollo \\ Santiago, Chile \\ https://orcid.org/0000-0002-7774-8354 \\ ximenaferrada@udd.cl
}

\begin{abstract}
RESUMEN
Actualmente, la infraestructura de salud pública en Chile, en sus distintas escalas, presenta avances significativos en la determinación de criterios de diseño en sustentabilidad. Sin embargo, es posible observar que durante su construcción no existen procesos de verificación estandarizados sobre aspectos de sustentabilidad y que la labor de la inspección técnica se centra en el cumplimiento administrativo de los contratos de construcción, más que en la verificación de los aspectos técnicos.

La presente investigación propone una lista de partidas y actividades críticas a fiscalizar en la construcción de hospitales para asegurar criterios sustentables en su operación. Luego, se realiza

una encuesta a profesionales que participan en el diseño, construcción y fiscalización de obras hospitalarias. Los resultados se jerarquizan con una metodología multicriterio (AHP), a partir de la cual se evidencia una preferencia en la envolvente térmica (20\%) e instalaciones térmicas y de ventilación (17\%). Finalmente, en base a las debilidades señaladas por los profesionales, se propone un proceso de control y seguimiento a estas partidas y actividades con un rediseño de la labor del Inspector Técnico de Obras.
\end{abstract}

Palabras clave

infraestructura sanitaria, inspección, sustentabilidad, metodología multicriterio (AHP)

\footnotetext{
ABSTRACT

Currently, the public health infrastructure in Chile, at its different scales, has made important advances in determining sustainability design criteria. However, it is possible to see that, during its construction, there are no standardized verification processes on sustainability aspects, and that the work of technical inspection focuses on the administrative compliance of construction contracts, rather than on checking

technical aspects. This research proposes a list of critical items and activities to supervise hospital construction, to guarantee sustainable criteria in their operation. A survey was also made to professionals involved in the design, construction, and supervision of hospital construction, ranking the results with a multi-criteria methodology (AHP), which showed a preference in the thermal envelope (20\%) and thermal and ventilation installations (17\%). Finally, based on the weaknesses stated by the professionals, a control and monitoring process of these items and activities is proposed, redesigning the work of the Worksite'

Technical Inspector.
} 


\section{INTRODUCCIÓN}

Criterios relacionados con la satisfacción o el bienestar de los equipos de trabajo profesionales, pacientes y administradores, en conjunto con la eficiencia de los recursos del edificio, deben considerarse durante todas las etapas de un proyecto de construcción, particularmente, en los edificios hospitalarios (Rodriguez, Svensson y Wood, 2020; Soliman-Junior et al., 2021). En ese sentido, se han desarrollado estándares sostenibles en edificios de salud, a través de distintas certificaciones como LEED, WELL o LBC (Allen et al., 2015), las que poseen un crédito llamado "comisionamiento", entendido como el conjunto de procesos que les permitiría realizar la fiscalización del proyecto en todas sus etapas (Lord, Noye, Ure, Tennant y Fisk, 2016). Además, en su aplicación, algunos autores han demostrado que un edificio certificado tiene una productividad adicional de 16\% (Vasquez et al., 2013), gracias a la mejora de la iluminación, control de la temperatura, mejora en la calidad del aire interior (Xuan, 2015) y reducción en 15\% del ausentismo (Vasquez et al., 2013).

Aun así, durante la operación del edificio no se puede afirmar que los hospitales que han sido certificados con puntajes más altos logran ser más eficientes que aquellos que consiguen menor puntuación (Golbazi y Aktas, 2016). Junto a lo anterior, los edificios de infraestructura pública en salud no siempre cumplen con los criterios sostenibles descritos en la fase de diseño y existen pocos estudios que analicen en detalle las razones de ello (Balali y Valipour, 2021).

Asimismo, durante la fase de construcción de la infraestructura en salud pública no existen procesos de verificación estandarizados de las directrices del proyecto en aspectos de sustentabilidad, lo que podría afectar el ciclo de vida sostenible de estas edificaciones. Los errores en la ejecución de las partidas críticas de la infraestructura de salud tienen diversas consecuencias durante la operación de una edificación (Castro, Mateus y Bragança, 2015), desde mantenciones correctivas excesivas y aumento del gasto público por energía extra de los sistemas energéticos (D’Amanzo, Mercado y Ganem Karlen, 2020), hasta malestar ambiental en las personas que utilizan el edificio, ya sea por sobrecalentamiento, sobreenfriamiento o por mala calidad del aire. En temas constructivos, dichas equivocaciones pueden conllevar aumento de costos de postventa por problemas de humedad o incorrecta impermeabilización, incorrecta ejecución de aislación o errores en las instalaciones sanitarias (CarreteroAyuso y García, 2018).

De este modo, las labores de Inspección Técnica - Inspección Fiscal de obras públicas en Chile son fundamentales para verificar que lo estipulado en el proyecto se cumpla una vez ejecutado. En concreto, la
Inspección Técnica posee dos objetivos principales: 1) inspeccionar las condiciones administrativas, referidas a aspectos financieros, plazos y normativas legales; y 2) inspeccionar las condiciones técnicas, referidas a la ejecución del proyecto para resguardar el cumplimiento contractual entre diseño y ejecución, y así asegurar la estabilidad, durabilidad y habitabilidad del proyecto (Ministerio de Vivienda y Urbanismo [MINVU], 2007). Sin embargo, ha quedado en evidencia que su labor se centra en el cumplimiento administrativo de los contratos de construcción más que en la verificación de los aspectos técnicos de los proyectos. Los principales problemas, en este contexto, están asociados a la incompatibilidad de los planos de diversas disciplinas, diferencias de criterio entre el personal de oficina y de campo, y a la baja retroalimentación en las metodologías de trabajo (Santelices, Herrera y Muñoz, 2019).

Un estudio de la Contraloría General de la República (Moscoso, 2017) indica que la observación de mayor relevancia en contratos de obra públicas de salud es el incumplimiento de aspectos técnicos, mientras que otros estudios académicos verifican que las falencias en los procesos de diseño son el principal factor que incide en los problemas que se desencadenan en las obras (Montiel-Santiago, Hermoso-Orzáez y TerradosCepeda, 2020). Esto refleja la necesidad de contar con protocolos y normas constructivas que se incorporen desde el inicio del diseño y que sean acompañadas de una efectiva inspección técnica de obra enfocada en la correcta funcionalidad de las características de los sistemas e instalaciones en establecimientos hospitalarios.

El presente estudio identifica las partidas críticas -conjunto de actividades o faenas de las etapas constructivas de un proyecto (Instituto Nacional de Normalización [INN], 1999a)- que deben ser inspeccionadas en la ejecución de una obra de salud y que impactan en su ciclo de vida sostenible, entre ellos, la calidad del ambiente interior y la eficiencia en el consumo energético o de agua durante la operación. En ese marco, se realizó, por una parte, un análisis de las actividades críticas de una partida, con el fin de identificar criterios sustentables de diseño que requiriesen ser asegurados para la fase de operación. Por otra parte, la información se validó mediante una encuesta realizada a profesionales que participan en el diseño, construcción y fiscalización de obras hospitalarias. Los resultados se analizaron con la metodología de priorización AHP para jerarquizar las partidas y actividades críticas y detectar las posibles debilidades que observan estos profesionales en su labor. Finalmente, en base a las preguntas abiertas de la encuesta, se propuso un proceso simplificado de seguimiento a partidas y actividades críticas capaz de utilizarse en todo el ciclo de un proyecto de infraestructura de salud pública con énfasis en la etapa de ejecución. 


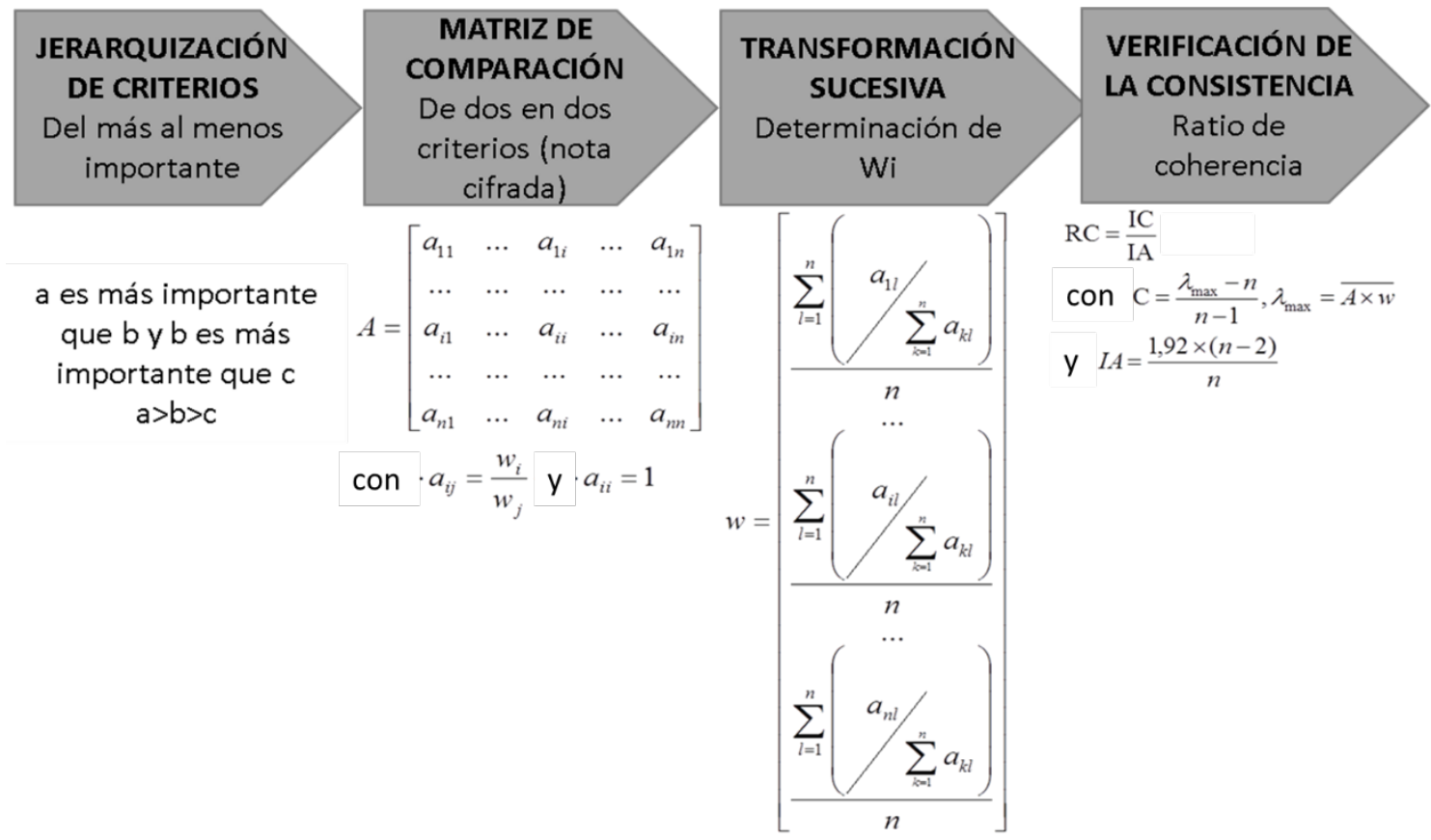

Figura 1. Etapas de la metodología AHP. Fuente: Elaboración de los autores a partir de Saaty $(1977 ; 2008)$.

\section{METODOLOGÍA}

Esta investigación plantea un proceso simplificado de inspección de hospitales, con énfasis en la ejecución. Para su exposición, el presente artículo se ha dividido en tres etapas.

En la primera fase, se presenta una descripción y un análisis del contexto de los establecimientos hospitalarios en Chile, en aras de entender cómo funciona su gestión, y se profundiza en la inspección técnica de las obras.

En la segunda etapa, se determinan las partidas y actividades críticas que se deben fiscalizar en el proceso de construcción de centros hospitalarios; labor que se alimenta de los requerimientos que componen la Certificación de Edificio Sustentable [CES] de Hospitales (Instituto de la Construcción [IC], 2017). Luego, se jerarquizan las partidas y actividades críticas a través de una encuesta aplicada a profesionales que participan en el diseño, construcción y fiscalización de obras hospitalarias.

El grupo de profesionales fue seleccionado considerando experiencia en el diseño, seguimiento e inspección directa de proyectos de salud de al menos 5 años. Los profesionales consultados fueron 9 arquitectos y 7 ingenieros, así como 10 inspectores técnicos.

La encuesta se desarrolló en tres partes. Primero, se debía responder la pregunta ¿cuáles de las partidas/actividades son más importante de fiscalizar durante la construcción de un hospital para asegurar que la ocupación/explotación del edificio cumpla con estándares sustentables? Para responder, se les pidió que eligieran, en pares, las partidas críticas más importantes a evaluar en la ejecución de proyectos hospitalarios y, en seguida, entre cada actividad crítica de cada partida. En segundo lugar, se consultaron opiniones respecto a los procesos de inspección, tales como: ¿qué dificultades se presentan en obra para fiscalizar las partidas o actividades anteriormente mencionadas? y ¿qué medidas se podrían implementar en obra para asegurar la correcta ejecución de las partidas y actividades? Por último, se les preguntó su opinión sobre la necesidad de apoyar la labor de la inspección técnica con la inclusión de otro profesional, y se les solicitó describir y/o comentar la labor que ejerce el inspector técnico. La segunda y tercera parte de la encuesta permitieron proponer un nuevo proceso de seguimiento y control durante la fase de construcción encaminado a asegurar los estándares sustentables definidos durante la fase de diseño.

Los resultados de la comparación de pares de alternativas de partidas y actividades se priorizaron a través de un método de priorización multicriterio, en el que se calcula un peso $\left(w_{i}\right)$ para cada alternativa presentada, realizando una comparación de dos por dos de todos los criterios. El método seleccionado fue el Proceso de Análisis Jerárquico (AHP), de Saaty (2008), por su gran popularidad para determinar la priorización en problemas muy variados como política, aspectos sociales, deseos personales, educación, industria o ingeniería (Valderrama-Ulloa y Puiggali, 2014; Darko et al., 2019; Emrouznejad y Marra 2017). Las diferentes etapas de la priorización de AHP se ilustran en la Figura 1.

En este punto, cada alternativa se compara con un par, a través de 5 niveles. Por ejemplo, se puede constatar 


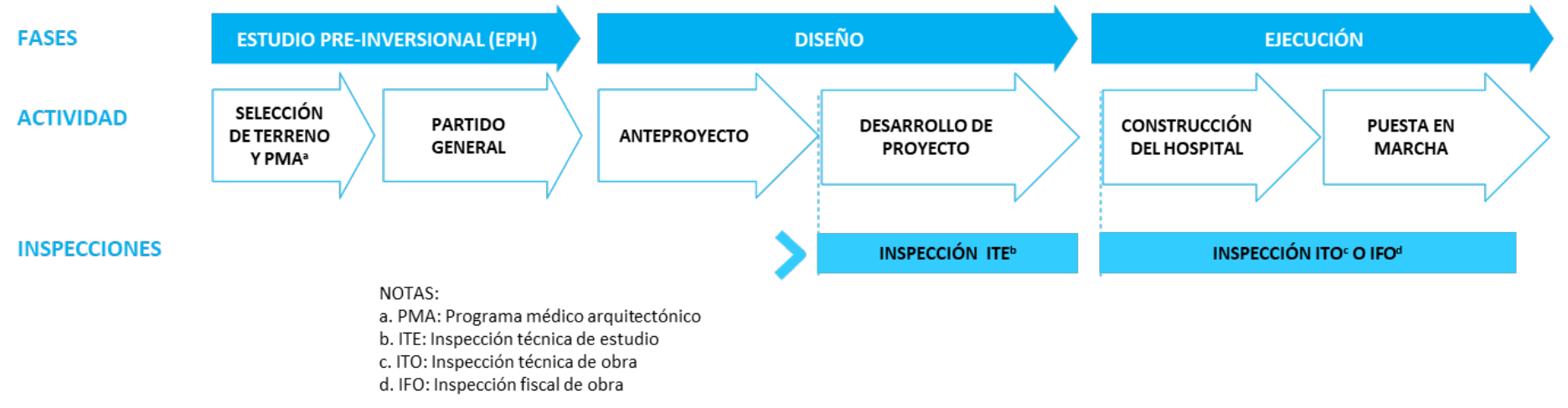

Figura 2. Esquema del proceso de construcción de un hospital. Fuente: Elaboración de los autores.

si la partida envolvente es "mucho más importante", "importante", "igual", "menos importante" o "mucho menos importante" que la partida terminaciones. Los resultados de la votación dan lugar a la matriz A de comparaciones pareadas de $\mathrm{n}$ alternativas. Después, se realizan transformaciones sucesivas para la determinación de $_{\mathrm{i}}$, que será el porcentaje de priorización. Posteriormente, se determina el nivel de consistencia (RC) para comprobar si el resultado es o no "razonable", con valores entre 0,20 a 0,10 .

En la tercera etapa y final, en base a los comentarios recibidos en la encuesta, se propone un proceso simplificado de inspección de hospitales, con énfasis en criterios mínimos de sustentabilidad a fiscalizar en las partidas y actividades que tienen relación con estos criterios durante la construcción de establecimientos hospitalarios.

\section{ANTECEDENTES}

\section{ESTABLECIMIENTOS HOSPITALARIOS}

El Ministerio de Salud de Chile (MINSAL) tiene, dentro de sus funciones, fijar las políticas y normas de inversión en infraestructura y equipamiento de los establecimientos públicos que integran las redes asistenciales (MINSAL, 2017). Para tales efectos, la Subsecretaría de Redes Asistenciales es la encargada de regular y supervisar el funcionamiento de las redes de salud a través del diseño de políticas, normas, planes y programas para su coordinación y articulación. En ese marco, cada proyecto debe cumplir con todos los requerimientos normativos que emanan desde el MINSAL en lo relativo a disposiciones sanitarias, calidad de la atención y satisfacción del usuario, como también en cuanto a los criterios y lineamientos con los cuales se desarrollarán los diseños. En general, el desarrollo de los proyectos hospitalarios se divide en las fases de estudio pre-inversional o pre-diseño, diseño (Figura 2) y ejecución. En la actualidad, las labores de inspección comienzan en la fase de diseño junto con el desarrollo del proyecto por equipos de inspección técnica de estudios, y una vez en la fase de ejecución se desarrolla la inspección técnica de obra.

Los hospitales en Chile corresponden a establecimientos de atención cerrada, que otorgan prestaciones de salud en régimen continuado de atención y que deben contar con recursos organizados de infraestructura, equipamiento y personal necesario para su funcionamiento permanente. En el presente, estos se configuran con un área de atención ambulatoria (consultas médicas, salas de procedimientos, unidades de apoyo diagnóstico terapéutico y emergencia), anexada y complementaria a la atención cerrada en donde se encuentran principalmente las hospitalizaciones.

En Chile, los hospitales son de gran relevancia para cubrir las necesidades de atención de salud de la población. No obstante, en materia de infraestructura de salud, el país se encuentra en una situación de déficit, lo cual se evidencia por la falta de camas de hospitalización por número de habitantes. El promedio de camas hospitalarias de los países integrados en la Organización para la Cooperación y el Desarrollo Económico, OCDE, era de 5,045 camas por cada mil habitantes en 2013. En el caso de Chile, se llega sólo a 2,18 camas por cada mil habitantes (Goyenechea, 2016). Esto ha generado, en la última década, la elaboración de planes de inversión en infraestructura hospitalaria que logren fortalecer la red asistencial existente. A pesar de los esfuerzos realizados, un diagnóstico llevado a cabo el año 2018 indica que aún el 80\% de los hospitales incumplen la norma actual de calidad de infraestructura, debido a la antigüedad de sus construcciones y que el $63 \%$ de los recintos cuenta con una edificación anterior a los años 80 (Sandoval y Leiva, 2018).

En temas relativos al ámbito de la sostenibilidad, desde mediados de la década de los 2000 el Ministerio de Salud de Chile ha incorporado criterios de sustentabilidad en los diseños de los establecimientos de salud. Los primeros esfuerzos se enfocaron en la eficiencia energética, estableciendo especificaciones de envolvente (muros y ventanas) con mejor comportamiento térmico, lo que permitió reducir demandas energéticas para el acondicionamiento térmico de los recintos, como 
también la incorporación de sistemas activos (sistemas de climatización e iluminación) más eficientes.

En 2017 entra en vigencia la Certificación CES Hospitales, sistema nacional voluntario de certificación de calidad ambiental y eficiencia energética para edificios de uso hospitalario. Su objetivo es evaluar, calificar y certificar la capacidad de un edificio de lograr niveles adecuados de calidad ambiental interior, con el uso eficiente de recursos y baja generación de residuos y emisiones (IC, 2017). El sistema de certificación CES se basa en el cumplimiento de requerimientos obligatorios, la obtención de puntaje, según el cumplimiento de cada requerimiento voluntario que la compone, y una verificación en obra en la etapa final del proceso de evaluación, la cual consiste en la recopilación de algunos antecedentes $y$, al menos, una visita de inspección efectuada por una Entidad Evaluadora. Actualmente, se fomenta su uso como metodología de trabajo para elegir los criterios sostenibles que deben cumplir los edificios hospitalarios (MINSAL, 2017).

\section{INSPECCIÓN DE LAS OBRAS HOSPITALARIAS}

En Chile no existe un procedimiento detallado para la inspección de obras hospitalarias. Si bien se cuenta con un Manual de Inspección Técnica de Obras (MINVU, 2007), su alcance radica en la inspección de viviendas sociales, el que, con el tiempo, se extendió a proyectos de pavimentación y áreas verdes (Moscoso, 2017).

Un estudio sobre observaciones y recomendaciones en la ejecución de contratos de obra pública, que realizó la Contraloría General de la República entre los años 2012 y 2015, concluyó que gran parte de estas observaciones tienen relación directa con la función que cumple la inspección técnica de obras, destacando el incumplimiento de la normativa técnica, el cual representaba un 27\% de los casos (Moscoso, 2017). También reveló que el 51\% de las observaciones negativas se vinculaban a establecimientos de salud y que este tipo de proyecto no incluía al inspector técnico en etapas tempranas del diseño, por lo que se generaban dos problemas principales: 1) uso deficiente del tiempo, dado que el inspector técnico era contratado o asignado sólo al inicio de la obra, debiendo invertir tiempo en estudiar el proyecto y todos los documentos administrativos que formaban parte de dicha obra; y 2) desaprovechamiento del aporte y la experiencia del inspector técnico de obra en etapas tempranas del proyecto, donde toda modificación tiene un impacto menor en los costos del mismo.

Otro estudio sobre Inspección Técnica de Obras (ITO) indica que hay un número importante de hechos y situaciones que dan origen a conflictos y dudas, no siempre atribuibles a una decisión equivocada del mandante o a la gestión deficiente del Contratista (Corporación de Desarrollo Tecnológico [CDT], 2011). Estos conflictos suelen tener su origen en algunas de las siguientes causas: proyectos que no cuentan con un adecuado nivel de detalle, lo que impide definir, con precisión y objetividad, las exigencias y estándares que deben ser verificados por la ITO; proyectos que no presentan eficiente coordinación entre sí, ni con las especialidades; no se aprecia con claridad los roles y responsabilidades que deben asumir los diferentes profesionales del proceso constructivo en su participación en las distintas etapas de los proyectos; tanto los análisis y las evaluaciones se deben apoyar en antecedentes descoordinados y de escasa confiabilidad; $y$, las indefiniciones, omisiones y errores que presentan las bases administrativas de los contratos dan origen a un importante número de conflictos en la relación entre las partes.

En base a lo descrito se propone un listado de partidas críticas relevantes a cumplir y verificar en el proceso constructivo (fase de ejecución, etapa de construcción de la Figura 2) de hospitales para asegurar la sustentabilidad en su operación.

\section{RESULTADOS Y DISCUSIÓN}

A continuación, se presentan los principales resultados del estudio, incluyendo la lista y definición de las partidas y actividades críticas relevantes para la construcción de hospitales sustentables, la jerarquía realizada por los profesionales encuestados, junto con recomendaciones para fortalecer la inspección técnica y un proceso simplificado de control y seguimiento con énfasis en la etapa de ejecución, que permita asegurar criterios y variables sustentables durante la operación.

\section{PARTIDAS Y ACTIVIDADES CRÍTICAS RELEVANTES PARA LA CONSTRUCCIÓN SOSTENIBLE DE HOSPITALES PÚBLICOS}

Para la elección de las partidas seleccionadas, primero se analizó cada una de las variables CES que tenían una relación con las partidas en obra gruesa o de terminación de un proyecto de construcción (INN, 1999b) y, luego, se seleccionaron las partidas en que la variable CES asociada tenía los mayores puntajes en la certificación. Finalmente, cada partida seleccionada se subdividió en actividades para que el Inspector Técnico de Obra, durante la etapa de construcción, pueda fiscalizar más detalladamente dicha partida. Así, la Tabla 1 muestra la relación variable CES con la partida en obra asociada, la lista de las partidas seleccionadas y actividades que se analizaron en esta investigación.

\section{JERARQUÍA DE PARTIDAS Y ACTIVIDADES CRÍTICAS}

Respecto a la consulta sobre "¿cuáles de las partidas es más importante fiscalizar durante la construcción de un hospital 


\begin{tabular}{|c|c|c|c|}
\hline Variable CES & $\begin{array}{c}\text { Partidas en obra } \\
\text { asociadas }\end{array}$ & Partidas seleccionadas & Actividades que la componen \\
\hline Confort visual - pasivo & Envolvente térmica & \multirow{3}{*}{ Envolvente } & \multirow{3}{*}{$\begin{array}{c}\text {-verificación del tipo de material y espesor del } \\
\text { aislante del: complejo cielo-cubierta, del muro } \\
\text { envolvente, de los pisos ventilados } \\
\text {-verificación del tipo de cristales y marcos en } \\
\text { ventanas } \\
\text { - verificación del tipo de material y } \\
\text { continuidad de aislación para evitar los } \\
\text { puentes térmicos } \\
\text { - verificación del tipo de material y ejecución } \\
\text { de protecciones solares } \\
\text { - verificación del tipos de materiales de la } \\
\text { barrera de vapor y de humedad }\end{array}$} \\
\hline Demanda de energía & Envolvente térmica & & \\
\hline $\begin{array}{l}\text { Hermeticidad de la } \\
\text { envolvente }\end{array}$ & Envolvente térmica & & \\
\hline Confort acústico & Aislación acústica & \multirow[b]{2}{*}{ Aislación acústica } & \multirow{2}{*}{$\begin{array}{c}\text { - verificación del tipo de material y espesor de: } \\
\text { elementos divisorios horizontales y verticales } \\
\text { - verificación de la hermeticidad de uniones y } \\
\text { juntas de: los elementos divisorios horizontales } \\
\text { y de los verticales } \\
\text {-controlar ruido y vibraciones: de los equipos, } \\
\text { de los ductos de extracción/inyección de aire, } \\
\text { de ascensores y montacargas } \\
\text {-verificar la aislación acústica en la red de } \\
\text { alcantarillado }\end{array}$} \\
\hline Ruido equipos & Aislación acústica & & \\
\hline $\begin{array}{c}\text { Calidad del aire - } \\
\text { pasivo }\end{array}$ & Terminaciones interiores & Terminaciones & $\begin{array}{c}\text { - verificar los revestimientos interiores } \\
\text { verticales, cielos, pavimentos y pinturas y } \\
\text { barnices sean los especificados }\end{array}$ \\
\hline Confort visual - activo & $\begin{array}{l}\text { Instalaciones de } \\
\text { iluminación y eléctricas } \\
\text { (confort visual) }\end{array}$ & \multirow{2}{*}{$\begin{array}{l}\text { Instalaciones de } \\
\text { iluminación y eléctricas }\end{array}$} & \multirow{2}{*}{$\begin{array}{l}\text { - verificar que las luminarias sean las } \\
\text { especificadas } \\
\text {-verificar que los sensores de iluminación e } \\
\text { interruptores sean los especificados }\end{array}$} \\
\hline Consumo de energía & $\begin{array}{l}\text { Instalaciones de } \\
\text { iluminación y eléctricas }\end{array}$ & & \\
\hline $\begin{array}{l}\text { Confort térmico - } \\
\text { activo }\end{array}$ & $\begin{array}{l}\text { Instalaciones térmicas } \\
\text { y ventilación (confort } \\
\text { térmico y ventilación) }\end{array}$ & \multirow{3}{*}{$\begin{array}{l}\text { Instalaciones térmicas y } \\
\text { de ventilación }\end{array}$} & \multirow{3}{*}{$\begin{array}{c}\text {-verificar el tipo, características y } \\
\text { funcionamiento del sistema de climatización y } \\
\text { agua caliente sanitaria (ACS) } \\
\text {-verificación de aislación en la red de } \\
\text { distribución de climatización y ACS, } \\
\text {-correcta instalación y funcionamiento de } \\
\text { termostatos de climatización, del caudal } \\
\text { de aire de ventilación, del tipo de filtros en } \\
\text { ventilación y/o extracción forzada } \\
\text {-verificar ubicación y cantidad de sensores de } \\
\text { CO2. }\end{array}$} \\
\hline $\begin{array}{c}\text { Calidad del aire - } \\
\text { activo }\end{array}$ & $\begin{array}{c}\text { Instalaciones térmicas y } \\
\text { ventilación }\end{array}$ & & \\
\hline Consumo de energía & $\begin{array}{c}\text { Instalaciones térmicas y } \\
\text { ventilación }\end{array}$ & & \\
\hline Consumo de energía & $\begin{array}{l}\text { Sistemas de Energías } \\
\text { Renovables No } \\
\text { Convencionales }\end{array}$ & \multirow{2}{*}{$\begin{array}{l}\text { Sistemas de Energías } \\
\text { Renovables no } \\
\text { Convencionales }\end{array}$} & \multirow[t]{2}{*}{$\begin{array}{c}\text {-verificar correcta instalación y verificación de } \\
\text { funcionamiento de paneles fotovoltaicos o } \\
\text { colectores solares térmicos }\end{array}$} \\
\hline ERNC & $\begin{array}{l}\text { Sistemas de Energías } \\
\text { Renovables No } \\
\text { Convencionales }\end{array}$ & & \\
\hline $\begin{array}{c}\text { Sistemas de agua } \\
\text { potable }\end{array}$ & Instalaciones sanitarias & Instalaciones sanitarias & $\begin{array}{c}\text {-verificar correcta instalación de grifería de } \\
\text { lavamanos y lavafondos según EETT } \\
\text {-verificar el tiempo de temporizadores de } \\
\text { grifería de lavamanos } \\
\text {-verificar correcta instalación de inodoros } \\
\text {-verificar correcta instalación de grifería de } \\
\text { tinas y duchas según EETT } \\
\text {-verificar el tipo y características del sistema de } \\
\text { tratamiento de dureza del agua }\end{array}$ \\
\hline
\end{tabular}




\begin{tabular}{|c|c|c|c|}
\hline Manejo de residuos & Gestión de los residuos & $\begin{array}{c}\text { Gestión de residuos de la } \\
\text { operación }\end{array}$ & $\begin{array}{c}\text {-verificar espacio adecuado para asegurar la } \\
\text { gestión de residuos. }\end{array}$ \\
\hline Riego & Paisajismo & \multirow{2}{*}{ Paisajismo } & \multirow{2}{*}{$\begin{array}{c}\text {-verificación de su existencia, y de que sea de } \\
\text { bajo consumo hídrico }\end{array}$} \\
\hline Paisajismo & Paisajismo & & \\
\hline Energía incorporada & Obra gruesa & Obra gruesa & $\begin{array}{c}\text {-verificar que materiales estructurales cuenten } \\
\text { con etiqueta ambiental }\end{array}$ \\
\hline Agua incorporada & Obra gruesa & Obra gruesa & $\begin{array}{c}\text {-verificar que materiales estructurales cuenten } \\
\text { con etiqueta ambiental }\end{array}$ \\
\hline $\begin{array}{l}\text { Diseño integrado de } \\
\text { anteproyecto }\end{array}$ & - & - & - \\
\hline
\end{tabular}

Tabla 1. Detalle de variables sustentables y su relación con partidas de obras asociadas con las partidas y actividades seleccionadas. Fuente: Elaboración de los autores.

\begin{tabular}{|c|c|c|c|c|c|c|c|c|c|}
\hline Jerarquización & \multicolumn{10}{|c|}{ E $>$ ITV $>$ T $=$ EnR $>$ IIE $>$ IS $>$ P $>$ AA $>\mathbf{R}$} \\
\hline Partidas & $\mathrm{E}$ & $\mathrm{AA}$ & $\mathrm{T}$ & $\mathrm{IS}$ & $\mathrm{IIE}$ & $\mathrm{ITV}$ & $\mathrm{EnR}$ & $\mathrm{P}$ & $\mathrm{R}$ \\
\hline wi (\%) & 20 & 6 & 13 & 10 & 11 & 17 & 13 & 7 & 5 \\
\hline$\lambda$ máx & 11.47 & IC & 0.31 & IA & 1.54 & RC & 0.20 & & \\
\hline
\end{tabular}

Tabla 2. Jerarquización de cada partida a fiscalizar en la etapa de construcción para asegurar que la ocupación/explotación del edificio cumpla con estándares sustentables. Fuente: Elaboración de los autores.

para asegurar que la ocupación/explotación del edificio cumpla con estándares sustentables?", se observa (Tabla 2) una preferencia de un $20 \%$ para la Envolvente (E), seguida de Instalaciones Térmicas y de Ventilación (ITV), con un 17\%, y de Terminaciones (T) y Sistemas de Energías Renovables no Convencionales (EnR), con un 13\%; de ahí que la consistencia fuera un 0.20. Por último, las tres partidas menos votadas fueron Paisajismo (P), Aislación Acústica (AA) y Gestión de Residuos (R), con un $7 \%$, $6 \%$ y $5 \%$, respectivamente. Instalaciones Sanitarias (IS) e Instalaciones de lluminación y Eléctricas (IIE) tuvieron una valoración muy parecida, del 10 y $11 \%$, respectivamente.

Si bien el método AHP se aplicó a todas las partidas, acá se grafican las dos partidas más votadas. Las respuestas referentes a priorización de las actividades a fiscalizar en la envolvente (la partida con mayor prioridad, 20\%) se separaron en dos grupos -lo indicado por los arquitectos y lo indicado por ingenieros e ITOs (Figura 3)-, debido a que existe una gran diferencia en las prioridades indicadas por cada grupo, lo que no ocurrió en las votaciones de las otras partidas o actividades. De esta forma, las 3 actividades más votadas por el grupo de ingenieros e ITOs fueron la correcta instalación de: aislante del muro envolvente (26\%), barrera de vapor y humedad (16\%) y pisos ventilados con aislante de complejo cielo-cubierta (15\%). Para el grupo de arquitectos las prioridades fueron: aislación complejo cielo-cubierta (25\%), aislante del muro de envolvente (15\%) y cristales y marcos de ventanas (14\%).

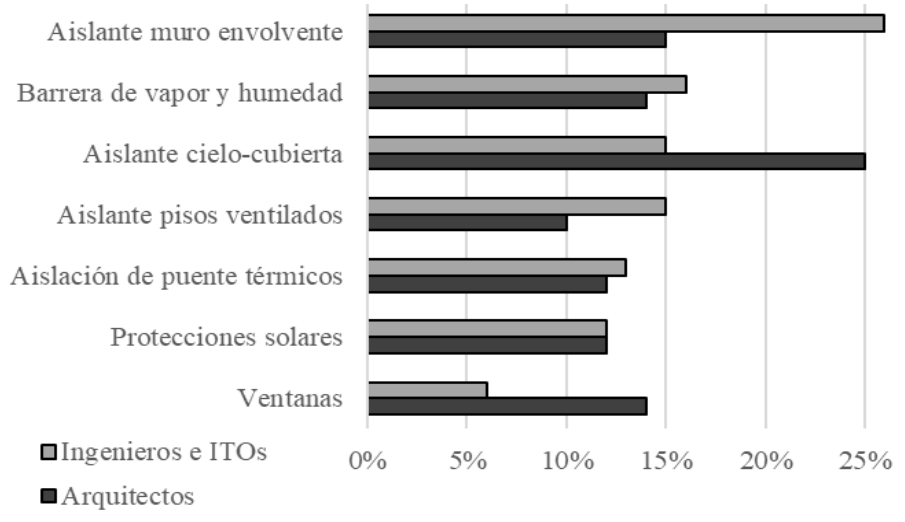

Figura 3. Priorización de los grupos de profesionales a las actividades de la partida envolvente. Fuente: Elaboración de los autores.

En el caso de las actividades que componen la partida de Instalaciones Térmicas y de Ventilación (la segunda más votada, 17\%) las 3 actividades más importantes para los profesionales fueron: funcionamiento de climatización y agua caliente sanitaria (ACS) (26\%), aislación de la red de climatización y ACS (22\%) y correcta instalación de termostatos de climatización (18\%). La menos importante a fiscalizar fue: filtros de ventilación y/o extracción forzada, que registró un $8 \%$ de las respuestas (Figura 4).

Adicionalmente, se consultaron opiniones respecto a los procesos de inspección técnica de las obras hospitalarias. En cuanto a las dificultades que se presentan en obra para inspeccionar las partidas o actividades previamente 


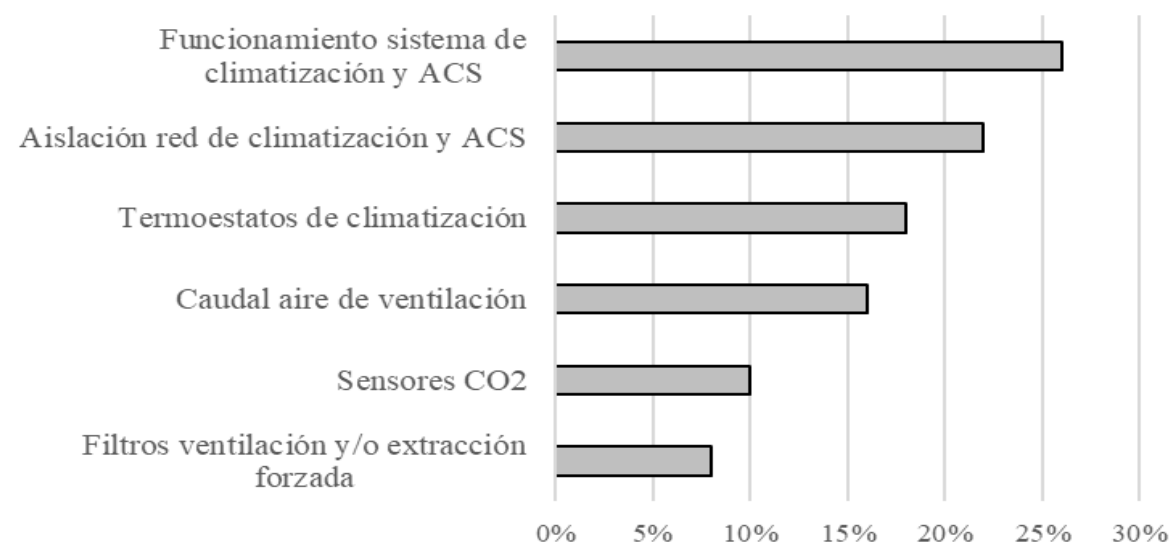

Figura 4. Priorización de los grupos de profesionales a las actividades de la partida Instalaciones Térmicas y de Ventilación. Fuente: Elaboración de autores.

priorizadas, el grupo de expertos indicó que las dos principales dificultades encontradas en obras son la falta de procedimientos (33\%) y que no existen cuerpos normativos que entreguen indicaciones (33\%). En tercer lugar, se reconoce una falta de conocimiento técnico (19\%). Otras dificultades mencionadas tienen relación con la calidad de los proyectos desde el diseño y la falta de autocontrol de las constructoras, partidas con especificaciones técnicas contradictorias y falta de reuniones de coordinación; todas con un $4 \%$ de las preferencias.

Respecto a las principales medidas que se podrían implementar en obra para asegurar la correcta ejecución de partidas, se mencionó el crear procedimientos de recepción en obra (46\%) y crear un cuerpo normativo que entregue indicaciones (42\%). Otras respuestas indican la necesidad de contar con proyectos fiscalizados por ITO, uniformar los antecedentes de los proyectos y contar con apoyo técnico especializado (todas con un $4 \%$ ).

Ante la consulta de incluir un nuevo profesional especializado que ayude en la fiscalización, el 100\% de los encuestados respondió afirmativamente. $Y$ algunos comentarios adicionales del grupo de expertos consultados señalaron tres temas principales que se pueden mejorar en la fiscalización de atributos sustentables en obras hospitalarias, los que se detallan a continuación:

El rol de los profesionales:

- un nuevo profesional especializado no solo debe aparecer al inicio de la obra, sino que debe acompañar, incluso, en el proceso de diseño coordinando a las especialidades e informando al mandante.

- es primordial el desarrollo colaborativo de las especialidades por profesionales competentes para lograr un alto nivel de eficiencia energética y sustentabilidad del edificio

- es necesario asegurar en el diseño la incorporación de los aspectos de eficiencia energética y sustentabilidad en las especialidades.
Procesos de comprobación:

- incorporar mejores herramientas tecnológicas para control del proyecto y especialidades.

- hacer exigibles procesos de comprobación de cumplimiento de lo especificado, o bien, comprobación previa de los estándares especificados.

- normalizar pautas de chequeo, las cuales puedan ser obligatorias en el contrato, con el objetivo de que las pautas no queden al arbitrio del mandante o contratista.

- se deben generar visitas más seguidas del visitador de obra.

- verificar en la puesta en marcha del edificio el rendimiento de los sistemas

- es necesario que la instalación de los distintos sistemas energéticos tenga sus procedimientos de ejecución, explicación teórica y lista de chequeo de aceptación con tolerancias aceptables.

- una vez ejecutada la obra, debe ser certificada y evaluar su cumplimiento mediante mediciones que sean parte del proceso de recepción de la obra.

Contexto normativo:

- falta desarrollo de normativa que entregue indicaciones para los procedimientos de fiscalización para estas partidas críticas de sustentabilidad.

- faltan normas nacionales, especialmente en el área de climatización.

- las normas internacionales no se utilizan, en parte, por la brecha que generan los idiomas.

En base a la jerarquización de partidas y actividades aquí presentada, los comentarios levantados sobre las dificultades que se presentan en obra para fiscalizarlas, las medidas que se podrían implementar en obra para asegurar la correcta ejecución y los tres temas destacados en las preguntas abiertas de los profesionales (rol de los profesionales, procesos de comprobación y contexto normativo), se propone en el siguiente apartado un nuevo proceso de seguimiento y control de las partidas y actividades que permitiría asegurar los estándares sustentables en la fase de ejecución del proyecto (Figura 2), una vez definidos durante la fase de diseño. 


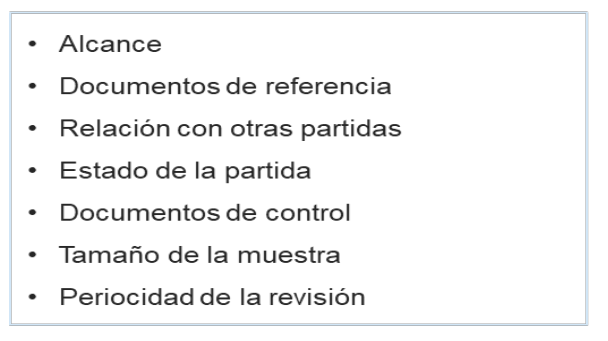

ESQUEMA DE PROCEDIMIENTO

Control en:

- Recepción del material o elemento,

- Inspección de la correcta ejecución

- Verificación del buen desempeñodel sistema.

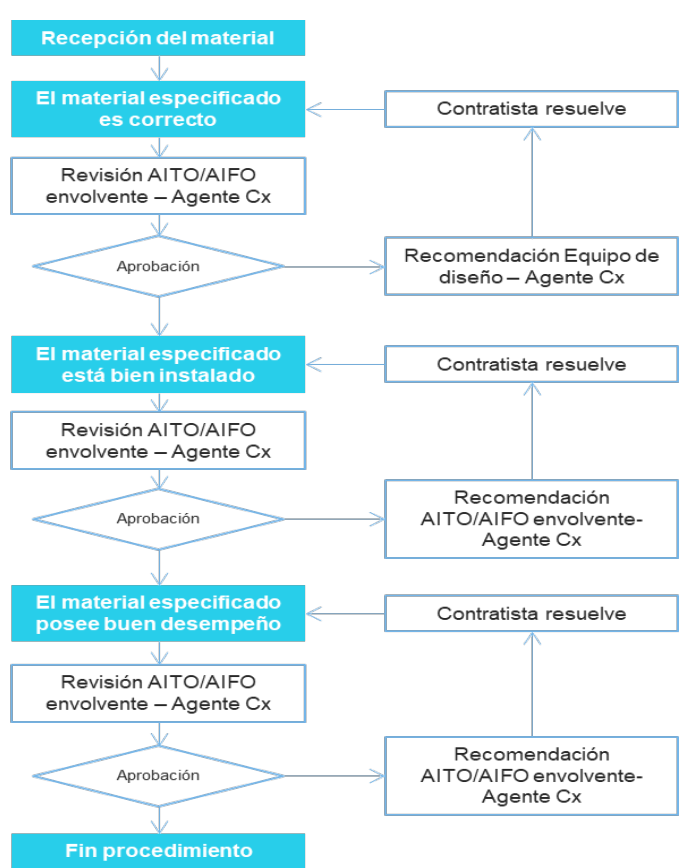

Figura 5. Diagrama de flujo para el seguimiento de la ejecución. Fuente: Elaboración de los autores.

\section{PROPUESTA DE INSPECCIÓN TÉCNICA}

La presente propuesta del proceso de control y seguimiento de la inspección técnica fue desarrollada considerando cualquier modalidad de contrato de obras hospitalarias. Se recomienda que en estos contratos la inspección técnica o fiscal comience desde el diseño y, así, facilitar la fiscalización, en la medida en que sea llevada a cabo desde una fase temprana por un mismo equipo especializado.

Esta propuesta de proceso de seguimiento y control reconoce las actuales labores que cumple la Inspección Técnica de Obras (ITO) y la Inspección Fiscal de Obras (IFO), sin embargo, gracias a la retroalimentación derivada de las preguntas abiertas de la encuesta aplicada, es posible atribuir a estas entidades nuevas responsabilidades y mayor participación en más etapas del proyecto. Asimismo, se prevé que los equipos de Asesores (AITO o AIFO) estén compuestos por especialistas con conocimiento específico y capacidad de efectuar tareas de apoyo a la realización del proceso de seguimiento y control de un proyecto de construcción, ya que en la actualidad solo poseen la certificación CES.

Para el desarrollo del mencionado proceso se requiere la figura de un coordinador denominado Agente de Inspección (Agente Cx), presente a lo largo del proyecto desde la fase de pre-diseño hasta la fase de operación y encargado de verificar y documentar que los sistemas e instalaciones hayan sido planificados, diseñados, instalados, probados, operados y mantenidos cumpliendo con los requerimientos de proyecto y tomando como prioridad las partidas y actividades relevadas en la encuesta de priorización.
En la Figura 5 se ilustra el flujo de este nuevo proceso de seguimiento y control del proyecto con énfasis en la fase de ejecución (Figura 2) y en la verificación del desempeño y funcionamiento de las instalaciones y sistemas constructivos. El flujo es esquemático, dado que su especificidad se trabajará en cada una de las partidas y actividades escogidas a partir del análisis multicriterio. Se compone también de un cuadro documental que se debe construir para cada proyecto - - , idealmente, que utilice el MINSAL en todos sus proyectos-, y un esquema de procedimiento que resalta los controles en la ejecución de la partida o actividad. Se termina con el diagrama en el que se detallan los profesionales que deben estar involucrados en el proceso.

\section{CONCLUSIONES}

Actualmente, el MINSAL desarrolla un extenso plan de inversiones en infraestructura de salud, planteando un gran desafío para este sector orientado a construir bajo parámetros de sustentabilidad y de mantener adecuadamente estas nuevas infraestructuras durante su operación.

Dependiendo del tipo de contrato para la ejecución de una obra hospitalaria, el flujo de información entre las distintas fases puede verse obstaculizado, debido a que distintos equipos realizan el diseño, la construcción y las fiscalizaciones en cada una de ellas, de forma independiente y sin el seguimiento apropiado. Por la complejidad que caracteriza a los establecimientos de salud, se requiere de procesos de verificación continuos 
y en todas las etapas, que aseguren el funcionamiento de los sistemas e instalaciones proyectadas.

Por otra parte, el proceso de levantamiento de información con expertos $y$, luego, su ponderación a través de la metodología AHP sobre la elección de partidas y actividades críticas para ser inspeccionadas en obras hospitalarias, permitió verificar la relevancia de cada una. Se evidenció que las partidas más relevantes son la Envolvente Térmica (20\%) y las Instalaciones Térmicas y Ventilación (17\%).

Los resultados de las encuestas dan cuenta de la necesidad de contar con normativas técnicas que entreguen procedimientos y estándares de fiscalización de los sistemas e instalaciones. Es necesario el desarrollo de proyectos coordinados y de mejor calidad desde la fase de diseño. Se reconoce el aporte de un profesional especializado que ayude en la fiscalización, que se incorpore desde fases tempranas del diseño. Además, muchas de las normativas a aplicar en los sistemas e instalaciones son traducciones de normas extranjeras (por falta de las propias nacionales) y su fiscalización posee una barrera idiomática. En este sentido, no basta con una traducción de normas sino, más bien, una institución dedicada a su elaboración aplicada al contexto nacional.

Un proceso de seguimiento y control también permitiría ver las repercusiones que tienen las decisiones tomadas en la obra a partir del diseño, ya sea por lo que se está exigiendo o por lo que ofrece el mercado. La inclusión temprana de un proceso oficial de seguimiento y control dará lugar a un grupo de lecciones aprendidas en las obras hospitalarias de las cuales disponer. La dificultad en su implementación se centraría tanto en la necesidad de formación profesional y calificación de Agentes Cx, como en la de normativas con protocolos y procedimientos específicos para cada partida, de modo similar a lo que hace el crédito de comisionamiento en las certificaciones medioambientales internacionales, incluyendo a las instalaciones y sistemas energéticos. Con base en los sistemas ya existente en Chile, este proceso permitirá, asimismo, ordenar, fiscalizar, asegurar y cumplir los requerimientos de la actual certificación CES Hospitales.

\section{AGRADECIMIENTOS}

Este trabajo se desarrolló bajo la tesis "Guía de inspección técnica en obra en criterios sostenibles para infraestructura en salud - Propuesta de una metodología para la inspección técnica de obras hospitalarias" del Magíster en Construcción Sustentable de la Pontificia Universidad Católica de Chile.

\section{REFERENCIAS BIBLIOGRÁFICAS}

Allen, J. G., Macnaughton, P., Laurent, J. G. C., Flanigan, S. S., Eitland, E. S. y Spengler, J. D. (2015). Green buildings and health. Current environmental health reports, 2(3), 250-258. DOI: https:// doi.org/10.1007/s40572-015-0063-y
Balali, A. y Valipour, A. (2021). Prioritization of passive measures for energy optimization designing of sustainable hospitals and health centres. Journal of Building Engineering, 35(2). DOI: https://doi.org/10.1016/J.JOBE.2020.101992

Carretero-Ayuso, M. J. y García Sanz-Calcedo, J. (2018). Analytical study on design deficiencies in the envelope projects of healthcare buildings in Spain. Sustainable Cities and Society, (42), 139-147. DOI: https://doi.org/10.1016/J. SCS.2018.07.004

Castro, M. de F., Mateus, R. y Bragança, L. (2015). A critical analysis of building sustainability assessment methods for healthcare buildings. Environment, Development and Sustainability, 17(6), 1381-1412. DOI: https://doi.org/10.1007/ S10668-014-9611-0

Corporación de Desarrollo Tecnológico [CDT] (2011). Inspección Técnica de Obras: una mirada al futuro de la calidad. Santiago de Chile: Ed. Cámara Chilena de la Construcción.

D'amanzo, M., Mercado, M. V. y Ganem Karlen, C. (2020). 10 preguntas de los edificios energía cero: revisión del estado del arte. Revista Hábitat Sustentable, 10(2), 24-41. DOI: http:// dx.doi.org/10.22320/07190700.2020.10.02.02

Darko, A., Chan, A. P. C., Ameyaw, E. E., Owusu, E. K., Pärn, E. y Edwards, D. J. (2019). Review of application of analytic hierarchy process (AHP) in construction. International journal of construction management, 19(5), 436-452. DOI: https://doi. org/10.1080/15623599.2018.1452098

Emrouznejad, A. y Marra, M. (2017). The state of the art development of AHP (1979-2017): a literature review with a social network analysis. International Journal of Production Research, 55(22), 6653-6675. DOI: https://doi.org/10.1080/0 0207543.2017 .1334976

Golbazi, M. y Aktas, C. B. (2016). Analysis of credits earned by LEED healthcare certified facilities. Procedia Engineering, 145, 203-210. DOI: https://doi.org/10.1016/j.proeng.2016.04.062

Goyenechea, M. (2016). Dificultades de la inversión en infraestructura pública de salud en Chile: concesiones y licitación pública. Recuperado de http://www.medwave.cl/link. cgi/Medwave/ Revisiones/Analisis/6444.act?ver=sindiseno.

Instituto de la Construcción [IC] (2017). Manual Certificación Edificio Sustentable CES-Hospitales. Evaluación y Calificación. Santiago de Chile: Instituto de la Construcción,

Instituto Nacional de Normalización (1999a). Norma chilena oficial NCh 1156/1. Of.1999: Construcción - Especificaciones Técnicas - Ordenación y designación de partidas. Parte 1: Generalidades. Santiago de Chile.

Instituto Nacional de Normalización (1999b). Norma chilena oficial NCh 1156/3. Of.1999: Construcción - Especificaciones Técnicas - Ordenación y designación de partidas. Parte 3: Obras de Construcción. Santiago de Chile.

Lord, S. F., Noye, S., Ure, J., Tennant, M. G. y Fisk, D. J. (2016). Comparative review of building commissioning regulation: a quality perspective. Building Research \& Information, 44(5-6), 630-643. DOI: https://doi.org/10.1080/09613218.2016.11819 55 
Ministerio de Salud (MINSAL) (2017). Orientaciones Técnicas para Diseño de Anteproyectos de Hospitales Complejos. Santiago: Gobierno de Chile, MINSAL Internal document working. Recuperado de https://www.minsal.cl/wp-content/ uploads/2018/02/ORIENTACIONES.pdf

Ministerio de Vivienda y Urbanismo [MINVU]. (2007). Technical Works Inspection Manual. Gobierno de Chile. Recuperado de https://www.minvu.cl/wp-content/uploads/2019/05/DS_N_85_ Manual_ITO_2007.pdf

Montiel-Santiago, F. J., Hermoso-Orzáez, M. J. y Terrados-Cepeda, J. (2020). Sustainability and energy efficiency: BIM 6D. study of the BIM methodology applied to hospital buildings. Value of interior lighting and daylight in energy simulation. Sustainability, 12(14), 1-29. DOI: https://doi.org/10.3390/SU12145731

Moscoso, P. (2017). Estudio base para el desarrollo de una metodología de inspección técnica de obras hospitalarias, Santiago, Chile. Tesis de magíster. Pontificia Universidad Católica de Chile.

Rodriguez, R., Svensson, G. y Wood, G. (2020). Assessing corporate planning of future sustainability initiatives in private healthcare organizations. Evaluation and Program Planning, 83. DOI: https://doi.org/10.1016/J.EVALPROGPLAN.2020.101869

Saaty, T. L. (1977). A scaling method for priorities in hierarchical structures. Journal of mathematical psychology, 15(3), 234-281.

Saaty, T. L. (2008). Relative measurement and its generalization in decision making why pairwise comparisons are central in mathematics for the measurement of intangible factors the analytic hierarchy/network process. RACSAM-Revista de la Real Academia de Ciencias Exactas, Físicas y Naturales. Serie A. Matemáticas, 102(2), 251-318.

Sandoval, G. y Leiva, L. (28 de julio de 2018). La obsolescencia de la red pública hospitalaria. La Tercera. Recuperado de https:// www.latercera.com/nacional/noticia/la-obsolescencia-la-redpublica-hospitalaria/260412/

Santelices, C., Herrera, R. y Muñoz, F. (2019). Problems in quality management and technical inspection of work. Revista Ingeniería de Construcción, 34(3), 242-251. DOI: https://doi.org/10.4067/ S0718-50732019000300242

Soliman-Junior, J., Tzortzopoulos, P., Baldauf, J. P., Pedo, B., Kagioglou, M., Formoso, C. T. y Humphreys, J. (2021). Automated compliance checking in healthcare building design. Automation in Construction, 129. DOI : https://doi.org/10.1016/J. AUTCON.2021.103822

Valderrama-Ulloa, C. y Puiggali, J.-R. (2014). Requerimientos de usuarios de la edificación traducidos en una metodología de apoyo a la decisión. Informes de la Construcción, 66(534), 1-11. DOI: http://dx.doi.org/10.3989/ic.12.106

Vasquez, E., Rola, S., Martins, D., Alves, L., Freitas, M. y Rosa, L. P. (2013). Sustainability in Civil Construction: application of an environmental certification Process (LEED) during the construction phase of a hospital enterprise-Rio de Janeiro/Brazil. International Journal of Sustainable Development and Planning, 8(1), 1-19. DOI : http://dx.doi.org/10.2495/SDP-V8-N1-1-19
Xuan, X. (2015). Effectiveness of indoor environment quality in LEED-certified healthcare settings. Indoor and Built Environment, 25(5), 786-798. DOI: https://doi.org/10.1177/1420326X15587564 